\title{
Doubling the quality factor of cantilevers in liquid through fluid coupling-based actuation
}

\author{
Stephane Leahy, Yongjun Lai \\ Department of Mechanical and Materials Engineering \\ Queen's University, Kingston, ON K7L 3N6 \\ Corresponding email: lai@queensu.ca
}

\begin{abstract}
Dynamic-mode cantilevers are a promising tool for real-time biosensing applications due to their high sensitivity and ability to perform label-free measurements. However, operating dynamic-mode cantilevers in liquid is challenging since viscous damping greatly reduces their quality factor and thus limit of detection. We reasoned through physical analysis that if the motion of the surrounding fluid is driven by an external force and not by the sensing cantilever itself, then the dissipative fluid force on the cantilever could be reduced and the quality factor of the cantilever could be increased. Here we demonstrate a new fluid coupling-based actuation method where one piezoelectric cantilever (directly excited) is used to excite another closely located cantilever (indirectly excited) through vibrations transferred through the surrounding medium. We performed measurements in several mediums, including air, water, ethanol, and acetone and observed that the viscosity of the medium influences the effectiveness of fluid coupling-based actuation. We also observed that fluid coupling-based actuation is more effective for the first bending mode of the cantilever, likely since fluid motion decays with distance from the tip of the directly excited cantilever. A significant result is that the indirectly excited cantilever has a quality factor that is double that of the directly excited one for the first bending mode in water. This method could improve the performance of dynamic-mode cantilevers operated in liquid.
\end{abstract}

\section{Introduction}

Dynamic-mode cantilevers are used for a variety of applications including measuring the mass, concentration, growth, and interaction of biological particles, and measuring the density and viscosity of fluids [1]. They are valued for their high sensitivity and ability to perform quantitative, label-free measurements. For biosensing applications, the main metric used to quantify their performance is the limit of detection [2]. This is defined as the lowest quantity of a substance that can be distinguished from the absence of that substance with a stated confidence interval. The limit of detection of dynamicmode cantilevers depends critically on their quality factor [3]. Thus, they demonstrate exquisite performance when measurements are performed offline in low viscous environments such as gases or vacuum chambers [4]. However, it is a significant challenge to operate them in real time in liquid, where strong viscous effects severely deteriorate their quality factor. Significant efforts have been made to improve their quality factor in liquid. Solutions include using larger cantilevers operated at high order resonant modes [5], using in-plane resonant modes [6], [7], implementing feedback loops [8], or incorporating a microchannel in the cantilever [9]. Using higher order or in-plane resonant modes is 
challenging because it is difficult to excite these modes due to the increased effective stiffness of the cantilever [10]. Feedback loops can improve the measured signal, but they also increase the thermomechanical noise of the system [3]. Incorporating a microchannel in a cantilever increases system complexity and limits sample throughput [9]. Alternatively, photothermal excitation has been shown to improve the quality factor and stability of the frequency response of AFM cantilevers in liquid [11]. It is effective since it drives cantilevers without adding distortion to their frequency response.

Here we demonstrate a new actuation method for cantilevers to improve the quality factor of their first resonant mode in liquid. A piezoelectric-excited cantilever (directly excited) is used to actuate another closely located cantilever (indirectly excited) through fluid coupling. We study the response of both cantilevers in several mediums to understand how fluid coupling-based actuation affects the quality factor of cantilevers. This method is promising for biosensing application because it uses the fundamental resonant mode of the cantilever, it increases the inherent quality factor of the cantilever, and it does not increase system complexity.

\section{Physics}

The physics describing the frequency response of cantilevers immersed in viscous fluids has been rigorously described [12]. The analysis is valid for the first few bending modes when the vibration amplitude is small, the fluid is incompressible, and the length of the beam greatly exceeds its width. The analysis is elegantly presented and validated with experimental measurements [13].

When a cantilever vibrates in a fluid, the fluid resists the motion of the cantilever. This resistance can be described as an external force per unit length acting on the cantilever and can be separated into two components: a dissipative force that is proportional to the cantilever velocity and an inertial force that is proportional to the cantilever acceleration. The fluid force can be written as [13]

$$
F_{\text {fluid }}=-g_{1} \dot{w}-g_{2} \ddot{w}
$$

where $w=w(x, t)$ is the cantilever deflection at a point $x$ along its length at time $t, g_{1}$ is the coefficient for the dissipative force, and $g_{2}$ is the coefficient for the inertial force. The coefficients can be calculated using the hydrodynamic function. The quality factor of a cantilever vibrating in a viscous fluid is

$$
Q_{\text {fluid }}=2 \pi f_{0} \frac{\sqrt{1+L g_{2} / m}}{L g_{1} / m}
$$

where $f_{0}$ is the resonant frequency of the cantilever, $L$ is the length, and $m$ is the mass. It is important to note here that the motion of the fluid is assumed to be caused entirely by the motion of the cantilever.

If the motion of the fluid is driven by an external force and not by the cantilever itself, then the fluid force and thus the quality factor of the cantilever will change. We would expect the quality factor of the cantilever to increase if the fluid is moving in phase with the cantilever. As shown in recent work, an acoustic wave in air can be used to excite a nanoscale fixed-fixed beam [14]. It was shown that the fluid force applied to the beam depends on the relative velocity between the beam and the fluid. In our fluidcoupling based actuation method, we use one cantilever to excite the fluid, which in turn excites the other cantilever. This should lead to an increase in quality factor, since less fluid will resist the motion of the cantilever. In addition, exciting the cantilever with moving fluid instead of a piezoelectric strip could 
lead to less distortion in the frequency response of the cantilever. This is the case with photothermal excitation [11], where the driving force is focused directly on the cantilever and not on other elements such as the substrate. The development of a physical model to describe our proposed fluid-coupling based actuation method will certainly require detailed simulation due to the complex relationship between the motion of both cantilevers and the surrounding fluid [15]. We leave this work for a future study and focus instead on a preliminary experimental analysis to introduce the concept.

\section{Methods}

\section{Design}

We used the PiezoMUMPs micromachining process [16] to fabricate two identical $200 \mu \mathrm{m}$ long cantilevers. We designed the cantilevers so that their free ends are facing each other and are separated by a $2.7 \mu \mathrm{m}$ gap [17] (see Figure 1). Each cantilever has a piezoelectric strip (aluminum nitride sandwiched between gold and doped silicon) at its fixed end and is suspended above a trench. Applying voltage across the piezoelectric strip (across the gold and the doped silicon on the cantilever) causes the cantilever to vibrate out of plane.

Figure 1 (Left) Isometric drawing of the cantilevers. The cantilevers, the anchored surfaces, and the applied electrical signal are identified. The dimensions are in $\mu \mathrm{m}$. The underlined dimension is not to scale. The material layers are color-coded: red is for silicon (doped at surface), blue is for aluminum nitride (piezoelectric material), and yellow is for gold. In the drawing, the piezoelectric strip is not included on the indirectly excited cantilever to emphasize the distinction between both cantilevers.

(Right) Optical image of the cantilevers.

\section{Measurements}

The chip hosting the cantilevers was secured to a microfluidic platform and the microfluidic platform was fixed to a probe station (Polytec MSA-400 Micro System Analyzer) with a magnet. Using a microscope, the two signal probes (ground and power) were lowered onto the electrode pads of the directly excited cantilever. Measurements were performed in different media, including air, acetone, water, and ethanol. For measurements in air, no intermediate steps were taken. For measurements in acetone, water, and ethanol, a droplet of the liquid was placed onto the surface of the chip and a glass slide mounted to a probe positioner was lowered above the chip to flatten out the droplet. In addition, for measurements in water, to ensure full immersion of the cantilevers, a syringe was connected to a port on the microfluidic platform and water was sucked through the trench below the cantilevers. In our previous work, we used a similar experimental setup and an detailed image of all the components are included there [18]. The directly excited cantilever was continuously excited with a periodic chirp signal with an amplitude of $3 \mathrm{~V}$. No excitation signal was applied to the indirectly excited cantilever. During this time, the vibration amplitude on the free end of each the directly excited cantilever and the indirectly excited cantilever was measured from 50 to $4000 \mathrm{kHz}$ using the vibrometer. The measured amplitudes were divided by the refraction index of the liquid (1.359 for acetone, 1.33 for water, and 1.361 for ethanol) to account for the lengthening of the optical path of the vibrometer laser when the target structure is immersed in a liquid ${ }^{1}$. The experimental mode shapes of each cantilever were also obtained by scanning the surface of each cantilever and building a two-dimensional point cloud with the vibrometer software.

\footnotetext{
${ }^{1}$ http://www.polytec.com/fileadmin/user_uploads/Applications/General_Vibrometry/LM_AN_VIB-G04_TRansparent_Fluids_2005_11_PDF_E.pdf
} 


\section{Results}

\section{Excitation in air}

Figure 2 shows the frequency response in air of both the directly excited cantilever and the indirectly excited cantilever. The frequency response of the directly excited cantilever has five significant peaks. Using point clouds, we can confirm that the peak at $258 \mathrm{kHz}$ is the first bending mode, the peak at $1237 \mathrm{kHz}$ is the second bending mode, and the peaks between the first and second bending mode are the first torsional, lateral, and longitudinal modes of the cantilever. This frequency response is typical for a cantilever. The frequency response of the indirectly excited cantilever has two significant peaks. The appearance of these peaks is surprising since no energy is directly applied to the indirectly excited cantilever. Again, using point clouds, we can confirm that the first peak is the first bending mode and the second peak is the second bending mode of the cantilever. In Supplementary Material, we include an animation of the first and second modes of the directly and indirectly excited cantilevers in air. These animations represent the experimental point clouds that were captured by the vibrometer software. We clearly observe the mode shapes of the cantilevers. Also, we see that both cantilevers are vibrating in phase with each other for both modes. Since, dissipative effects are small, this suggests that the indirectly excited cantilever is being driven by inertial effect of the vibrating fluid.

Figure 2 Comparison of the frequency response in air of the directly excited cantilever with that of the indirectly excited cantilever.

The frequency response of both cantilevers indicates that a portion of the energy applied to the directly excited cantilever is being transferred to the indirectly excited cantilever. The energy transferred is significant, since it causes the indirectly excited cantilever to vibrate with an amplitude only about one order of magnitude lower than that of the directly excited cantilever. To determine the mechanism of energy transfer, we did three tests. First, to eliminate the possibility of electrostatic energy transfer across the free ends of the cantilevers, we connected the doped silicon layer of both cantilevers to a common ground and verified that their frequency responses remained unchanged. Second, to eliminate the possibility of vibrations being transmitted from one cantilever to the other through the chip substrate, we performed measurements in a large area surrounding the anchor points of the cantilevers and verified that this area had no significant vibration. Third, to see if the energy was being transferred through fluid coupling, we performed measurements in several mediums, including air, water, ethanol, and acetone. Here we observed significant changes in the frequency response of both cantilevers. We discuss these results in the subsequent sections.

The peaks of the indirectly excited cantilever are aligned with those of the directly excited cantilever. We expect both cantilevers to have the same resonant frequencies because they have the same geometry. The peaks for the first torsional, lateral, and longitudinal modes of the indirectly excited cantilever are essentially absent. This can be partly attributed to the low amplitude of the peaks of the directly excited cantilever. However, it also suggests that fluid coupling-based actuation is dependent on the resonant mode. We expect this dependence, since the distribution of the kinetic energy along the directly excited cantilever varies with the mode order and mode type (bending, torsional, longitudinal and lateral). For the first mode, most of the kinetic energy is concentrated at the free end of the directly excited cantilever, close to the free end of the indirectly excited cantilever. However, for higher order 
modes, the kinetic energy is distributed along the directly excited cantilever, at points much farther away from the indirectly excited cantilever.

\section{Excitation in water}

Figure 3 shows the frequency response in water of the directly excited cantilever and the indirectly excited cantilever. Again, using point clouds, we can confirm that the peaks are the first and second bending modes of the cantilevers. In Supplementary Material, we include an animation of the first and second modes of the directly and indirectly excited cantilevers in water. These animations represent the experimental point clouds that were captured by the vibrometer software. We see that in water, unlike in air, the cantilevers are not vibrating in phase. This demonstrates that the cantilevers have different velocities relative to each other and thus relative to the surrounding fluid. While we can't measure the phase difference between the cantilevers and the liquid, we can infer that the directly excited cantilever is vibrating out of phase with the liquid [13] and the indirectly excited cantilever is vibrating nearly in phase with the liquid. This would enable the indirectly excited cantilever to have a higher quality factor, since it would experience less fluidic damping. As shown in Figure 3, the resonant frequencies and peak amplitudes of both cantilevers are significantly lower in water than in air. This is expected, since the higher density of water increases mass loading on the cantilevers and the higher viscosity increases damping. At the micrometer scale, viscous effects dominate over inertial effects and lead to significant damping and thus a large reduction in the quality factor of resonators [12].

Figure 3 Comparison of the frequency response in water of the directly excited cantilever with that of the indirectly excited cantilever.

We observe that the drop in peak amplitude of the indirectly excited cantilever relative to that of the directly excited cantilever is much lower here for the first resonant mode, when the surrounding medium is water, than when the surrounding medium is air (see Figure 2). This indicates that the surrounding medium influences how effectively the directly excited cantilever actuates the indirectly excited cantilever. This also confirms that fluid coupling is the mechanism through which the indirectly excited cantilever is actuated. In addition, we see that the relative drop in peak amplitude of the indirectly excited cantilever is much more important for the second resonant mode than it is for the first resonant mode. This supports our previous conclusion that fluid coupling-based actuation is mode dependent. When we measured even higher order bending modes past $1500 \mathrm{kHz}$, fluid coupling-based actuation became far less effective.

\section{Quality factor in several mediums}

To quantify the influence of the surrounding medium on the effectiveness of fluid coupling-based actuation, we measured the quality factor of both cantilevers in air, acetone, water, and ethanol. The quality factor is defined as the energy stored in a resonator relative to the energy loss. It is calculated using the resonance parameters provided by the vibrometer (Polytec MSA-400 Micro System Analyzer) software. The software fits a single harmonic oscillator model to a targeted frequency band in the measured frequency response. The results are listed in Table 1. 
Table 1 Quality factor of the first and second bending modes of the directly excited cantilever and the indirectly excited cantilever in several mediums.

\begin{tabular}{|l|l|l|l|l|l|}
\hline Medium & $\begin{array}{l}\text { Viscosity, } \\
\boldsymbol{\mu P a ~ s}\left(\mathbf{2 0}^{\circ} \mathbf{C}\right)\end{array}$ & $\mathbf{Q}_{\mathbf{1}}$ direct & $\mathbf{Q}_{\mathbf{1}}$ indirect & $\mathbf{Q}_{\mathbf{2}}$ direct & $\mathbf{Q}_{\mathbf{2}}$ indirect \\
\hline Air & 18.1 & 753.20 & 869.50 & 288.13 & 103.78 \\
\hline Acetone & 316 & 11.24 & 21.82 & 35.10 & 40.45 \\
\hline Water & 1002 & 7.85 & 17.95 & 28.25 & 34.16 \\
\hline Ethanol & 1095 & 5.39 & 12.75 & 18.47 & 23.27 \\
\hline
\end{tabular}

We see that the quality factors of both cantilevers decrease as viscosity increases. This is expected since an increase in viscosity should lead to an increase in energy loss, especially at the micrometer scale. We also note that the quality factor of the indirectly excited cantilever is higher than that of the excited cantilever in all cases except one (second resonant frequency in air). In general, we expect the indirectly excited cantilever to have higher quality factors since it is not exposed to electronic noise introduced by the piezoelectric strip and its phase is better matched with that of the vibrating fluid. The discrepancy for the second resonant frequency in air could be attributed to measurement error of the quality factor due to the appearance of multiple peaks (see Figure 2 at $1300 \mathrm{kHz}$ ). To better visualize the results, we calculated the quality factor ratio, which is defined as the quality factor of the indirectly excited cantilever divided by that of the directly excited one for a given resonant mode (see Figure 4). These results were obtained using the method described in the section Measurements. We included measurements from two chips to show the repeatability of the results. The second bending mode of the second chip was too weakly actuated for us to extract the quality factors.

As shown in Figure 4, the quality factor ratio for both the first and second bending modes follows the same trend: it increases significantly at lower viscosity and increases slightly at higher viscosity. The levelling off of the trend at higher viscosity (> $400 \mu \mathrm{Pa}$ s) could be attributed to a saturation of the peak amplitude of the indirectly excited cantilever. The peak amplitude of the indirectly excited cantilever can approach that of the directly excited cantilever as viscosity increases, but it cannot surpass it, because there is energy loss in the fluid. Moreover, the quality factor ratio for the second bending mode does not significantly surpass 1 . This indicates that fluid coupling-based actuation is much more effective for the first bending mode than it is for the second mode. As mentioned previously, the first bending mode of the directly excited cantilever likely generates higher fluid particle motion around the indirectly excited cantilever, leading to better actuation.

Figure 4 Quality factor ratio (quality factor of the indirectly excited cantilever divided by that of the directly excited one) as a function of viscosity for the first and second bending modes.

\section{Conclusion}

In conclusion, fluid coupling-based actuation is a method to double the quality factor of the first resonant frequency of microscale cantilevers in viscous fluids. For fluid coupling to be effective, the cantilevers need to be separated by a small gap, on the order of a few microns. Since the sensitivity of dynamic-mode cantilevers is related to their quality factor, fluid coupling-based actuation could be used the improve the performance of these devices. This method should be as effective, if not more effective, at smaller scales, where viscous effects become even more important. In future work, it would be 
interesting to study the effect of the gap size and the cantilever layout (cantilevers side-by-side versus facing each other) on the effectiveness of fluid-based actuation and see how well this method works for applications in biosensing or density and viscosity measurement.

\section{References}

[1] A. F. Payam, W. Trewby, and K. Voïtchovsky, "Simultaneous viscosity and density measurement of small volumes of liquids using a vibrating microcantilever," pp. 1492-1498, 2017.

[2] R. Mutharasan, M. Alvarez, and A. González, Biosensors and Biodetection: Methods and Protocols. Humana Press, 2009.

[3] J. Tamayo, "Study of the noise of micromechanical oscillators under quality factor enhancement via driving force control," J. Appl. Phys., vol. 97, no. 4, 2005.

[4] B. Ilic, "Attogram detection using nanoelectromechanical oscillators," J. Appl. Phys., vol. 95, no. 7, p. 3694, 2004.

[5] B. N. Johnson, "Biosensing using piezoelectric-based cantilever-like micro-electromechanical systems," ProQuest, 2013.

[6] Y. Tao et al., "Resonant cantilever sensors operated in a high-Q in-plane mode for real-time bio/chemical detection in liquids," Sensors Actuators B Chem., vol. 157, no. 2, pp. 606-614, Oct. 2011.

[7] W. Pang, L. Yan, H. Zhang, H. Yu, E. S. Kim, and W. C. Tang, "Femtogram mass sensing platform based on lateral extensional mode piezoelectric resonator," Appl. Phys. Lett., vol. 88, no. 24, pp. 3-5, 2006.

[8] M. Kucera et al., "Lock-in amplifier powered analogue Q-control circuit for self-actuated selfsensing piezoelectric MEMS resonators," Microsyst. Technol., vol. 20, no. 4-5, pp. 615-625, 2014.

[9] T. P. Burg et al., "Weighing of biomolecules, single cells and single nanoparticles in fluid," Nature, vol. 446, no. 7139, pp. 1066-1069, 2007.

[10] B. N. Johnson, H. Sharma, and R. Mutharasan, "Torsional and lateral resonant modes of cantilevers as biosensors: Alternatives to bending modes," Anal. Chem., vol. 85, no. 3, pp. 17601766, 2013.

[11] A. Labuda et al., "Photothermal excitation for improved cantilever drive performance in tapping mode atomic force microscopy," Microscopy and analysis, no. April, 2014.

[12] J. E. Sader, "Frequency response of cantilever beams immersed in viscous fluids with applications to the atomic force microscope," J. Appl. Phys., vol. 84, no. 1, p. 64, 1998.

[13] C. Vančura, I. Dufour, S. M. Heinrich, F. Josse, and A. Hierlemann, "Analysis of resonating microcantilevers operating in a viscous liquid environment," Sensors Actuators, A Phys., vol. 141, no. 1, pp. 43-51, 2008.

[14] R. Miles and J. Zhou, "Sound-Induced Motion of a Nanoscale Fiber," J. Vib. Acoust., vol. 140, no. 1, p. 011009, 2017.

[15] C. Intartaglia, L. Soria, and M. Porfiri, "Hydrodynamic coupling of two sharp-edged beams vibrating in a viscous fluid," Proc. R. Soc. A Math. Phys. Eng. Sci., vol. 470, no. 2162, pp. 
20130397-20130397, 2013.

[16] A. Cowen, G. Hames, K. Glukh, and B. Hardy, "PiezoMUMPs Design Handbook," 2013.

[17] S. Leahy and Y. Lai, "A gap method for increasing the sensitivity of cantilever biosensors," J. Appl. Phys., vol. 122, no. 6, 2017.

[18] S. Leahy and Y. Lai, "A cantilever biosensor exploiting electrokinetic capture to detect Escherichia coli in real time," Sensors Actuators B Chem., vol. 238, pp. 292-297, 2017. 


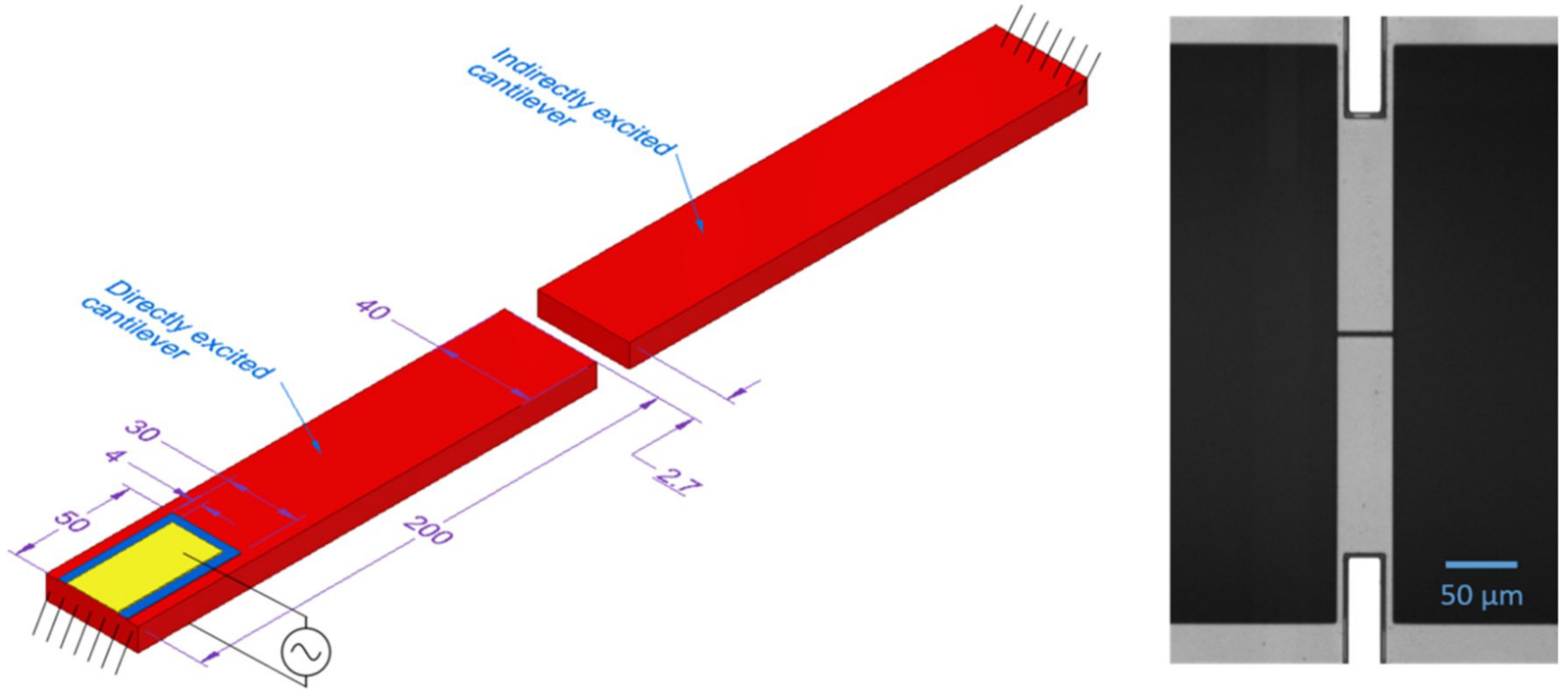




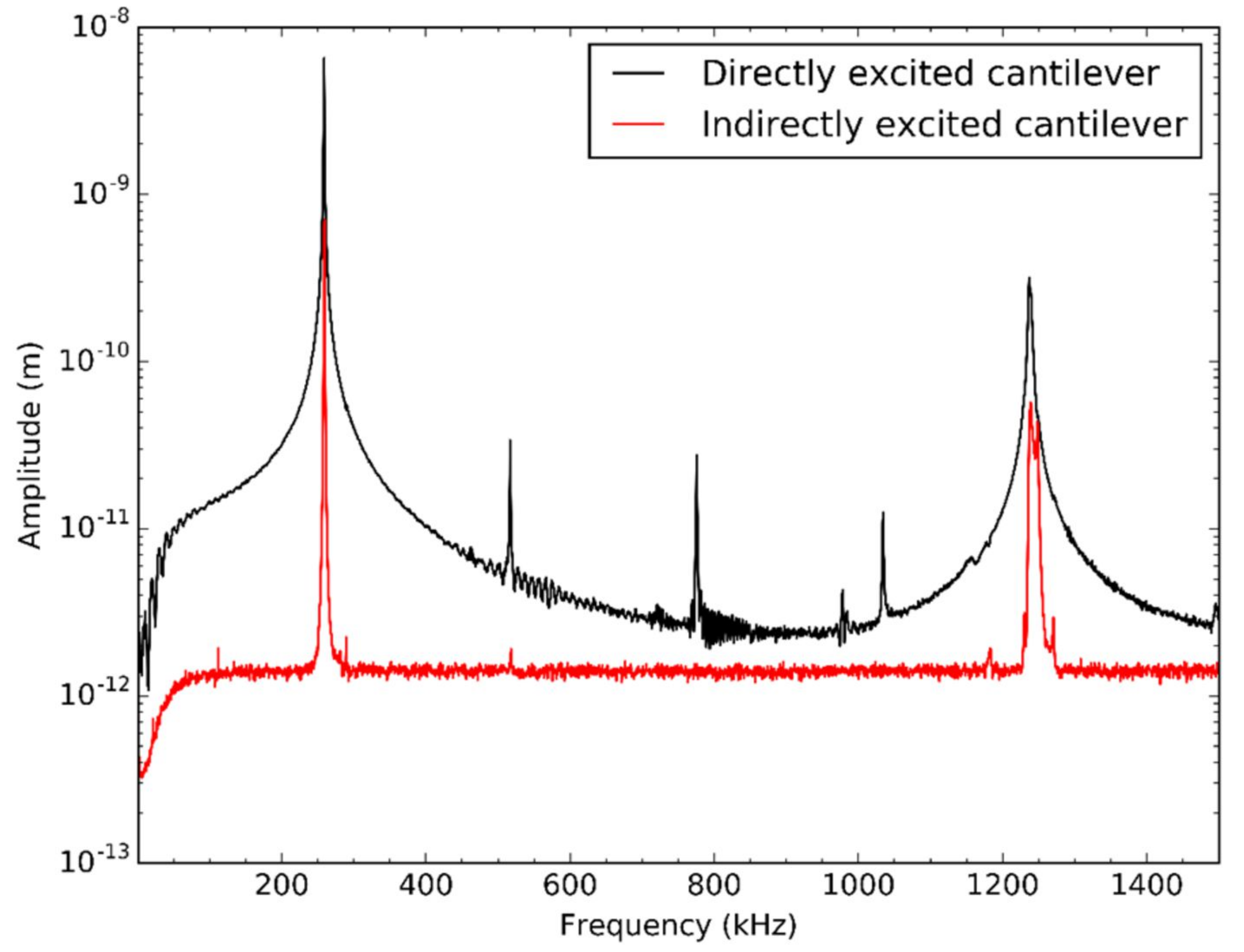




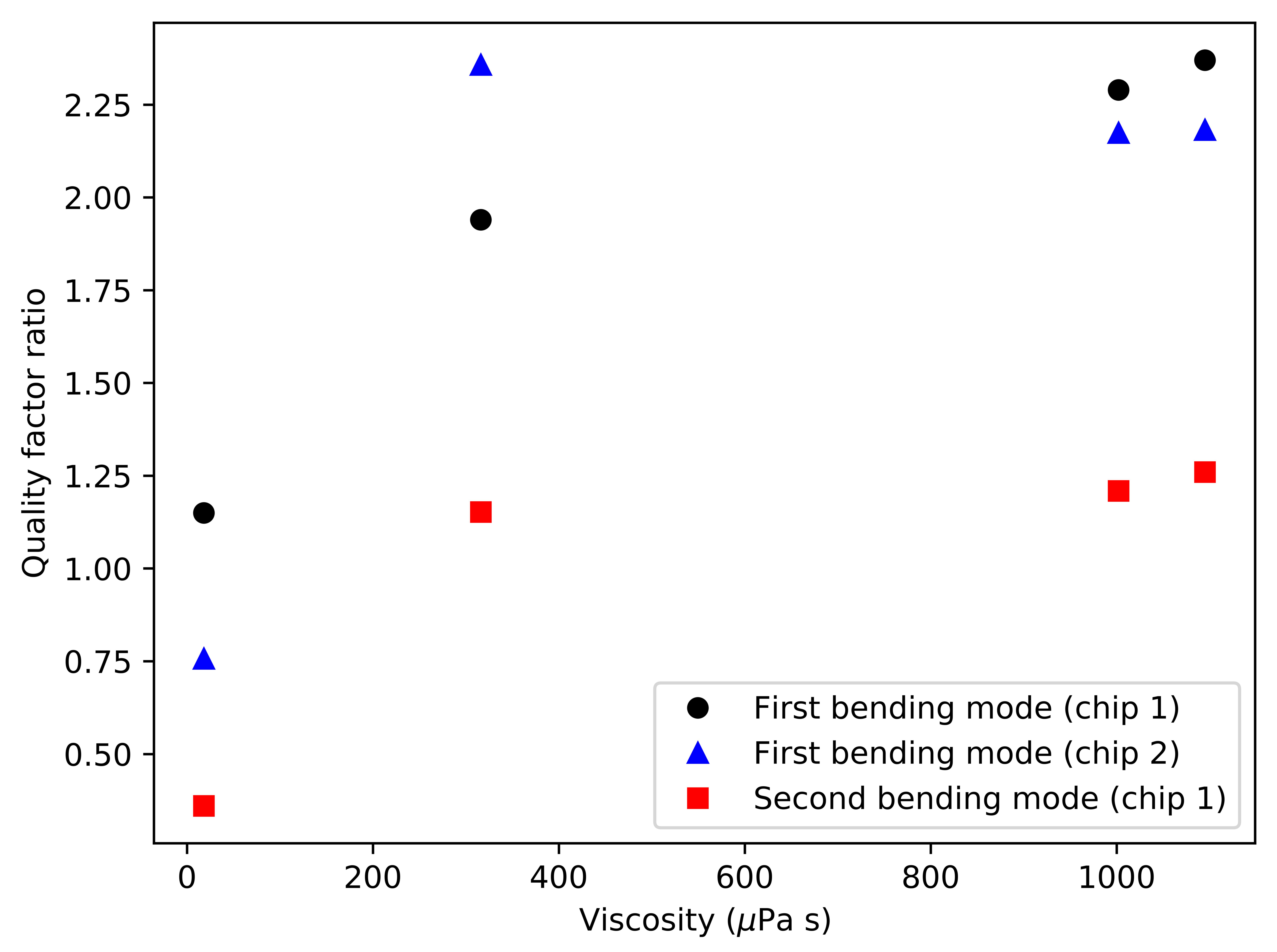

